\title{
Experimental Research on Thermocapillary-Buoyancy Migration Interaction of Axisymmetric Two Drops by Using Digital Holographic Interferometry
}

\author{
Shuoting Zhang ${ }^{1,2} \cdot$ Li Duan $^{1} \cdot$ Qi Kang ${ }^{1}$
}

Received: 6 June 2017 / Accepted: 29 November 2017 / Published online: 27 December 2017

(C) Springer Science+Business Media B.V., part of Springer Nature 2017

\begin{abstract}
The migration and interaction of axisymmetric two drops in a vertical temperature gradient is investigated experimentally on the ground. A silicon oil is used as the continuous phase, and a water-ethanol mixture is used as the drop phase, respectively. The migration and interaction of two drops, under the combined effects of buoyancy and thermocapillary, is recorded by a digital holographic interferometry measurement in the experiment to analyse the velocities and temperature distribution of the drops. As a result, when two drops migrate together, the drop affects the other drop by perturbing the temperature field around itself. For the leading drop, the velocity is faster than the one of the isolated drop, and the maximum of the interfacial temperature distribution is larger than the one of the isolated drop. For the trailing drop, the velocity is slower than the one of the isolated drop, and the maximum of the interfacial temperature distribution is less than the one of the isolated drop. The influence of the dimensionless initial distance between the drop centres to the drop migration is discussed in detail in this study.
\end{abstract}

Keywords Interfacial tension · Thermocapillary migration · Drop · Interaction

$\begin{array}{ll}\text { Nomenclature } \\ \mathrm{R} & \text { Drop radius, } \mathrm{m} \\ \mu & \text { Dynamic viscosity of the continuous phase, } \mathrm{Pa} \mathrm{s} \\ \rho & \text { Density of the continuous phase, } \mathrm{kg} \mathrm{m}^{-3} \\ \lambda & \text { Thermal conductivity of the continuous phase, } \\ & \mathrm{W} \mathrm{m}^{-1}{ }^{\circ} \mathrm{C}^{-1} \\ \sigma_{\mathrm{T}} & \text { Temperature coefficient of the interfacial tension, } \\ & \mathrm{N} \mathrm{m}^{-1}{ }^{\circ} \mathrm{C}^{-1} \\ \nu & \text { Kinetic viscosity, } \mathrm{m}^{2} \mathrm{~s}^{-1} \\ \Gamma & \text { Temperature gradient, }{ }^{\circ} \mathrm{C} \mathrm{m}^{-1} \\ \mathrm{~g} & \text { Gravitational acceleration, } \mathrm{ms}^{-2} \\ \mu^{\prime} & \text { Dynamic viscosity of the drop phase, Pa s } \\ \rho^{\prime} & \text { Density of the drop phase, } \mathrm{kg} \mathrm{m}^{-3}\end{array}$

Qi Kang

kq@imech.ac.cn

1 National Microgravity Laboratory, Institute of Mechanics, Chinese Academy of Sciences; School of Engineering Science, University of Chinese Academy of Sciences, Beijing 100190, China

2 China Nuclear Power Engineering Co., Ltd., Beijing, China $\lambda^{\prime} \quad$ Thermal conductivity of the drop phase, $\mathrm{W} \mathrm{m}{ }^{-1}{ }^{\circ} \mathrm{C}^{-1}$

$\sigma \quad$ Interfacial tension, $\mathrm{N} \mathrm{m}^{-1}$

$\kappa \quad$ Thermal diffusivity, $\mathrm{m}^{2} \mathrm{~s}^{-1}$

\section{Introduction}

When drops are placed in the immiscible continuous phase liquid possessing a temperature gradient, temperature variations can be expected to rise at the interface. For most fluid systems, the interfacial tension becomes decreased with increasing temperatures. The drops will migrate towards warm side. The migration is driven by the interfacial tension, and it is addressed as a thermocapillary migration.

Young et al. (1959) were the first to investigate the thermocapillary migration of bubbles in liquids on the ground. They observed small bubbles migration in pure liquids, and held the bubbles stationary or driven the bubbles downwards against buoyancy. After the pioneering work of Young et al., many investigations of the thermocapillary 
migration of drops in liquids were performed by theoretical analysis, numerical simulation and experiment.

The first theoretical study on two drops was carried out by Anderson (1985). With the method of reflections, the dynamics of two spherical droplets were analysed. The result was that the interaction between two droplets driven by thermocapillary migration was weaker than the one driven by gravitational force. The axisymmetric thermocapillary motion of two spherical drops in a constant applied temperature gradient along their line of centres was analysed by Keh and Chen (1990, 1992). The nearcontact thermocapillary motion of two drops was analysed by Loewenberg and Davis (1993). The asymptotic formula for the pairwise migration velocity, contact force, and the relative and individual drop velocities were presented for small size ratios were derived. They studied the thermocapillary-driven relative motion represented a more efficient coalescence mechanism than the gravity-driven relative motion. The axisymmetric motion and deformation of two viscous drops were investigated by Zhou and Davis (1996). It was found that the interactions between two drops had a stronger effect on the smaller of the two drops.

A numerical simulation of the thermocapillary motion of an interacting pair of drops was published by Lavrenteva and Nir (2003). The axisymmetric motion and the thermal wake interaction of two drops with the combined effects of gravity and thermocapillary was considered. They found that the thermal wake behind the leading drop had an influence on the temperature distribution of the trailing drop interface. The induced change in the speed of the trailing drop was large with its speed when isolated. Frolovskaya et al. (2006) studied the influence of the Marangoni effect on the drag force and the rate of heat transfer from the drops translating at given velocity. Numerical simulations of the thermocapillary motion of a pair of two-dimensional and three-dimensional fully deformable drops were presented by the front-tracking method (Nas and Tryggvason 2003; Nas et al. 2006). Yin et al. (2011) observed that the arrangement of the drops directly influences their migration and interaction, and the motion of one drop was mainly determined by the disturbed temperature field because of the existence of the other drop. Unequal drop sizes in the axisymmetric model was investigated by Yin and $\mathrm{Li}$ (2015). The effect of the ratio of the two drop radii, their initial distance apart, and non-dimensional numbers on the interaction were studied. They revealed the thermal wake behind the leading drop plays an important role in drop interaction.

The thermocapillary migration of a single water drops in the butyl benzoate was investigated by Hähnel et al. (1989). Their experimental results had a systematic deviation from the YGB linear theory, which was attributed to the convective transport inside and outside the drop ignored by the linear theory. The experiments were conducted on the ethyl salicylate drops migrating in the diethylene glycol by Barton and Subramanian (1989). Their results were consistent with the YGB linear theory qualitatively. Rashidnia and Balasubramaniam (1991) investigated the thermocapillary migration in drops of a vegetable oil immersed in the silicon oil. The liquids had equal densities at $15{ }^{\circ} \mathrm{C}$ to provide a simulation of the microgravity condition. Their experimental results followed the linear theory predictions qualitatively. However, the drop migrated farther away from the density-matched temperature compared to linear theory predictions. The drop migration was studied for middle Reynolds numbers by Xie et al. (1996). The experimental results shown drop migration velocities depended on the drop diameters, and were less than the YGB linear theory predicted. The temperature distribution around the drop during the thermocapillary migration and a thermal wake existed behind the drop was observed (Zhang et al. 2016). Some microgravity experiments for the thermocapillary migration of drops in the space shuttle have been performed. These experiments were conducted by Balasubramaniam et al. (1996) and Hadland et al. (1999) to investigate the thermocapillary migration of drops under reduced gravity. The researchers observed that the drop velocity against the Marangoni number.

The thermocapillary migration of single drops in liquids was subject to many experimental investigations. The experimental investigation of the thermocapillary migration of two bubbles or drops in liquids is, however, more difficult and thus performed less. The interaction between two bubbles was observed in the space experiment (Kang et al. 2008). In addition, Balasubramaniam et al. (1996) performed experiments on the thermocapillary motion of an interacting pair of drops. A small leading drop, which itself appeared unaffected in its motion, can significantly influence the motion of a larger trailing drop. It was conjectured that this was a consequence of the thermal wake behind the leading drop. The migration and interaction of two drops with different diameters in matrix liquid were observed by Kang et al. (2006). When two drops approached each other, the effect of the larger drop on the smaller one was significant, whilst the effect of the smaller one on the larger was weak.

However, amongst the experimental results mentioned above, few studies have studied the temperature distribution of the drops interface and the continuous phase liquid. Also, none of these studies has been investigated the coupling between temperature field and thermocapillary migration of two drops in the experiment. We present here experiment data revealing the coupling between temperature field and thermocapillary migration of two drops. As this is an important factor which will have far-reaching consequences 
for understanding of the mechanisms of thermocapillary migration, it is the focus of this present study.

The thermocapillary-buoyancy migration interaction of the axisymmetric two drops in a vertical temperature gradient has been investigated experimentally on the ground. The aim of the present work is to observe the interaction of two drops, and to provide quantitative temperature distribution data. To achieve this goal, digital holographic interferometry is used to record the interaction of two drops. The advantage of the present experimental study is the digital holographic interferometry which is used to measure the temperature field. The digital holographic interferometry is a non-destructive, non-invasive, in-situ, and full-field measurement. The temperature distribution of the interface and the surrounding of the drops during the interaction are visually studied. The coupling between temperature field and migration of two drops is examined. Also, the influence of the dimensionless initial distance between the drop centres to the interaction of two drops are discussed in detail.

\section{Experimental Setup and Procedure}

\section{Experimental Apparatus}

As shown in Fig. 1, there is a rectangular cell with a cavity $70 \mathrm{~mm}$ in length and $40 \mathrm{~mm} \times 40 \mathrm{~mm}$ in cross section. The test cell contained the continuous phase liquid. The top and bottom walls are two aluminium plates, and the four-sided wall is $8.0 \mathrm{~mm}$ thick optical glass. The top wall of the test cell is heated by electric heating film and the bottom wall is cooled by Peltier element, in order to maintain the temperature gradient. Two thermocouples are fixed into the top and bottom walls, and then connected with EUROTHERM 3504 controllers, in order to control the temperature differences between the two walls.
The digital holographic interferometry system is shown in Fig. 2, which is based on a double-exposure holographic interferometry method. Because of the advantages of a non-destructive, non-invasive, in-situ, and full-field measurement, digital holography is applied in the present experiment for measuring the temperature distribution of the liquid (Duan et al. 2008; Kang et al. 2004; Zhang et al. 2012). Digital holographic interferometry is an ideal method for the quantitative measurement of the temperature distribution.

As shown in Fig. 3, experimental setup for measuring the temperature is based on digital holographic interferometry. A laser beam from a fibre-coupled laser is split into two parts by an optical fibre coupler, which generates the object and reference waves, respectively. Then, the object and reference waves are collimated by the lens. The object wave passes through the liquid field whose the temperature change resulted in the corresponding change of the refractive index. Accordingly, the phase distribution of the object wavefront changes. A $4 \mathrm{f}$ system is introduced in the object beam path, to reduce the beam size comparable to the CCD target placed opposite to the beam splitter. And then, the object wave interferes with the reference wave, after transmission through the beam splitter, thereby forming the digital holograms carrying the information of the liquid field. The corresponding complex amplitude distributions of the object wave are numerically reconstructed from the holograms. Finally, the corresponding optical path difference of the object wave through the liquid field is obtained.

\section{Test Liquids}

In this study, $30 \mathrm{cSt}$ silicon oil and water-ethanol mixture (72.7-27.3 by weight) are selected as the continuous phase and the drop phase. The silicon oil and water-ethanol mixture are immiscible, and the densities of two liquids are
Fig. 1 Schematic diagram of the experimental apparatus

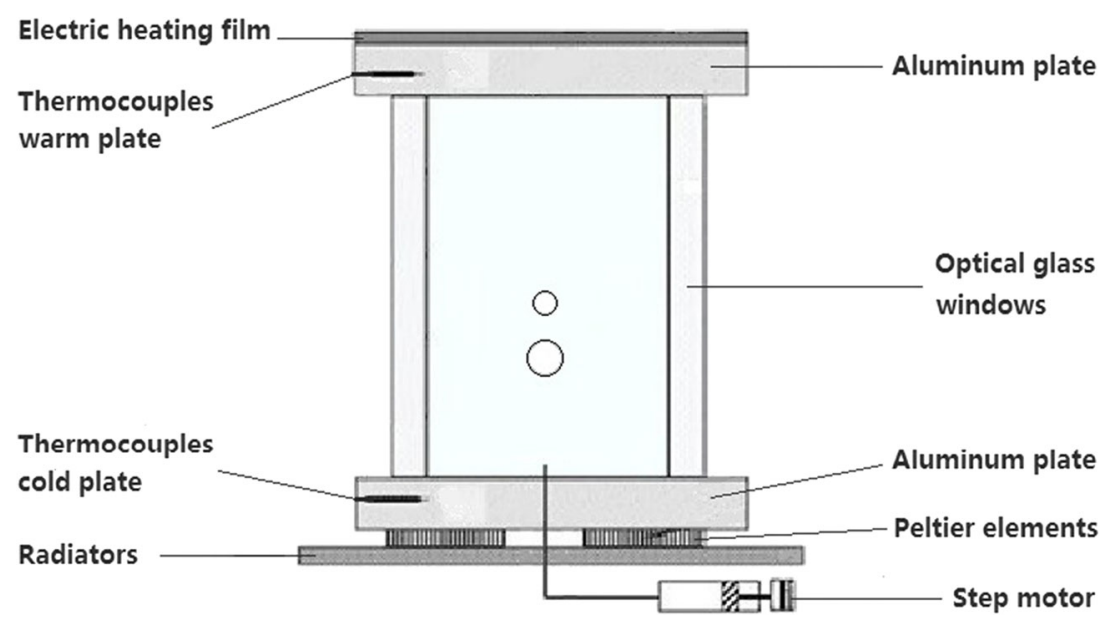


Fig. 2 Experimental apparatus

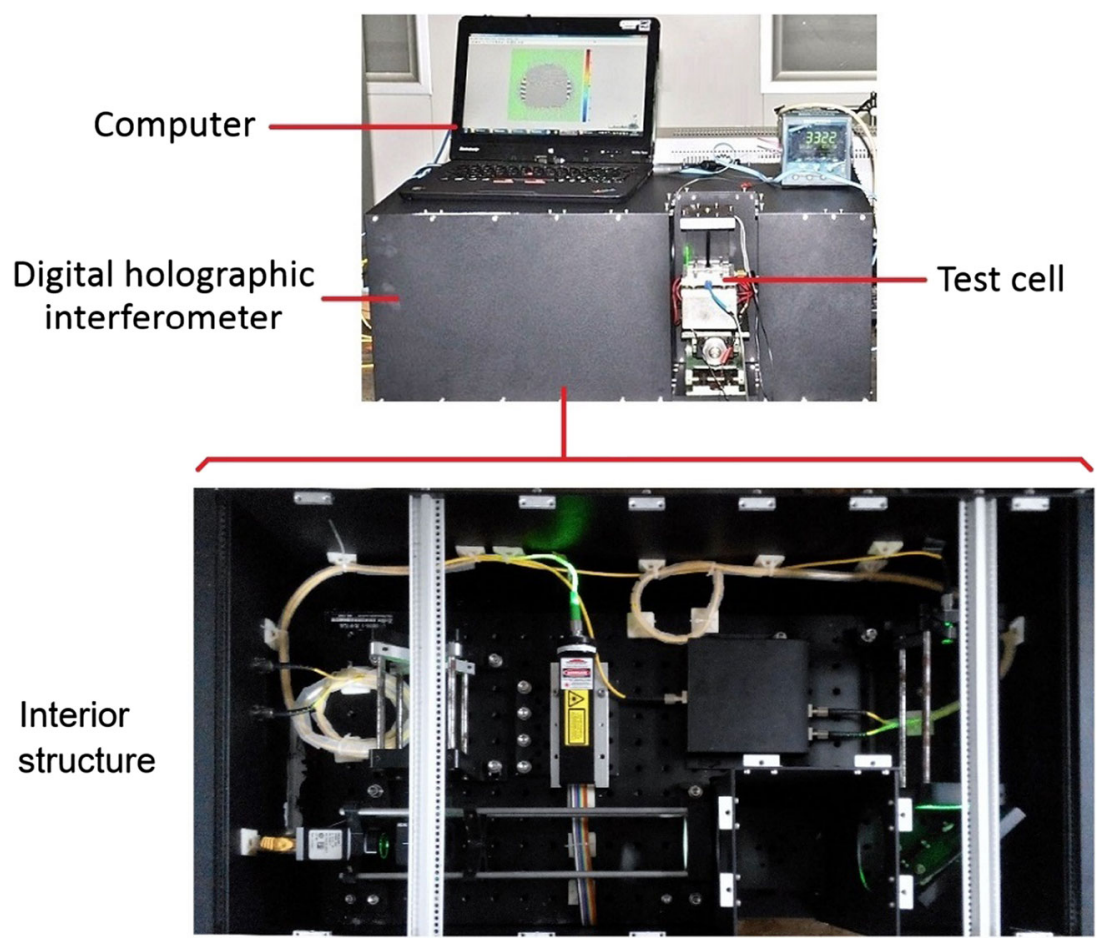

nearly equal at $25.0{ }^{\circ} \mathrm{C}$. The physical property parameters of the liquid system used in the experiment are listed in Table 1. Due to the fact that the temperature ranged between $15.0^{\circ} \mathrm{C}$ and $40.0^{\circ} \mathrm{C}$ in the experimental area, the data of the density variances with temperature are displayed in Table 2.

\section{The Physical Model}

Figure 4 shows the schematic diagram of the two drops migration. In this study, the two aligned arrangement drops are axisymmetric. The direction of the temperature gradient is along the $\mathrm{z}$-axis. The centre line of the two drops is the symmetry axis $(r=0) . R_{1}$ is the radius of the leading drop and $R_{2}$ is the radius of the trailing drop. The radius of the trailing drop is selected as the reference length, and $\Lambda$ is the radius ratio of the leading drop to the trailing drop. $\mathrm{H}$ is the distance between the drop centres and $\mathrm{H}_{0}$ the initial distance. $\mathrm{S}=\mathrm{H} / \mathrm{R}_{2}$ is the dimensionless distance between the drop centres and $\mathrm{S}_{0}=\mathrm{H}_{0} / \mathrm{R}_{2}$ the dimensionless initial distance. $\theta$ is defined as the angel from the interface to the drop centre. $\theta=0$ corresponds to the forward stagnation of the drop, and $\theta=\pi$ to the rear stagnation of the drop. $\mathrm{t}(\theta)$ represents the actual interfacial temperature distribution.

The reference speed $V_{0}$ is defined by the balance of the thermocapillary force and viscous force:

$V_{0}=\frac{\left|\sigma_{T}\right| \Gamma R}{\mu}$

In regards to the thermocapillary flow, the relative strength of the inertial forces to the viscous forces is measured by the dimensionless Reynolds number Re, defined as follows:

$$
R e=\frac{\rho V_{0} R}{\mu}
$$

Fig. 3 Experimental setup for measuring the temperature based on digital holographic interferometry

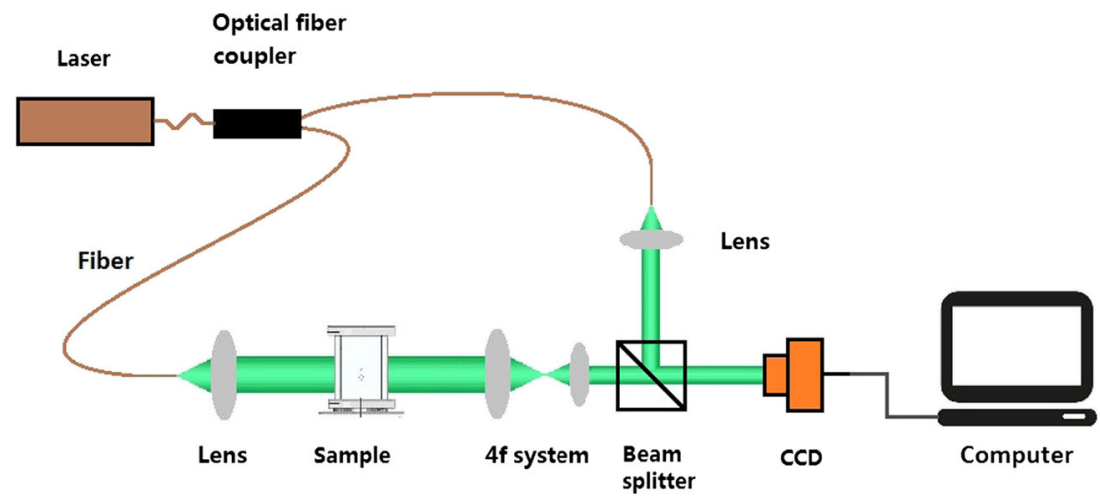


Table 1 Physical property parameters of the two liquids used for the experiments at $25.0^{\circ} \mathrm{C}$

\begin{tabular}{lllll}
\hline Fluid & $\nu\left(\mathrm{m}^{2} \mathrm{~s}^{-1}\right)$ & $\rho\left(\mathrm{kg} \mathrm{m}^{-3}\right)$ & $\lambda\left(\mathrm{W} \mathrm{m}^{-1}{ }^{\circ} \mathrm{C}^{-1}\right)$ & $\sigma_{\mathrm{T}}\left(\mathrm{N} \mathrm{m}^{-1}{ }^{\circ} \mathrm{C}^{-1}\right)$ \\
\hline $30 \mathrm{cSt}$ & $3.0 \times 10^{-5}$ & 955.0 & 0.151 & $-8.63 \times 10^{-5}$ \\
Water-ethanol mixture & $2.8 \times 10^{-6}$ & 955.0 & 0.41 & \\
\hline
\end{tabular}

The dimensionless Marangoni number Ma could then describe the ratio of the convective transport to the thermal conduction in the thermocapillary flow, defined as follows:

$M a=\frac{V_{0} R}{\kappa}$

The experimental data are compared with the YGB linear theory, which is used as a guideline. The YGB linear theory migration velocity of the drops is given by:

$\mathrm{V}_{Y G B}=\frac{2\left|\sigma_{T}\right| R \lambda \Gamma}{\left(2 \mu+3 \mu^{\prime}\right)\left(2 \lambda+\lambda^{\prime}\right)}+\frac{2\left(\rho-\rho^{\prime}\right) g R^{2}\left(\mu+\mu^{\prime}\right)}{3 \mu\left(2 \mu+3 \mu^{\prime}\right)}$

\section{Experimental Procedure}

The cell is filled with $30 \mathrm{cSt}$ silicone oil, and then the temperature gradient of the continuous phase liquid is established in the cell. After $2 \mathrm{~h}$, when the steady state conditions are achieved, the small drop is injected first into the cell, and the large drop is injected later. The migration interaction of two drops is recorded by digital holographic interferometry.

The digital holographic interferometry system is used to obtain the perturbed temperature distribution during the drops migration process. Due to the migration interaction of two drops in the continuous phase liquid, the refractive index has an axisymmetric change. The charge coupled device camera records a series of digital holograms of the liquid field in the initial and temperature variation states caused by the drops migration, which carries the refraction index and the temperature distribution information of the liquid field in different states, respectively. Then, the corresponding wrapped phase distributions images are numerically reconstructed from the digital holograms. By comparing the reconstructed complex amplitude of the object wave in the different states, the change of the optical path of the object wave is obtained. Then, the change of the refractive index of the liquid field is calculated from the holograms.

Table 2 Densities $\left(\mathrm{kg} \mathrm{m}^{-3}\right)$ at various temperatures

\begin{tabular}{llllll}
\hline Fluid & $15.0{ }^{\circ} \mathrm{C}$ & $20.0{ }^{\circ} \mathrm{C}$ & $25.0{ }^{\circ} \mathrm{C}$ & $30.0{ }^{\circ} \mathrm{C}$ & $35.0^{\circ} \mathrm{C}$ \\
\hline $30 \mathrm{cSt}$ & 963.3 & 959.4 & 955.0 & 950.6 & 946.4 \\
Water-ethanol mixture & 961.2 & 958.1 & 955.0 & 951.9 & 948.9
\end{tabular}

\section{Coupling Reconstruction of Two-Dimensional Field and Axisymmetric Field}

It should be noted that in regards to the drops migration interaction, the arrangement of the drops is aligned and axisymmetrical. Thus, the distribution of the refractive index change and the temperature change resulting from the drops migration is axisymmetrical, and is obtained by the onion-peeling algorithm (Dasch 1992).

The distribution of the refractive index change is only a function of the radius. Therefore, the corresponding optical path difference of the object wave through the liquid field caused by the refractive index change can be expressed as follows:

$\delta_{l}=\int_{l} \Delta n(r) d s$

Then, the line integral can be expressed as:

$\delta_{l}=2 \int_{r_{l}}^{r_{0}} \frac{\Delta n(r)}{\sqrt{r^{2}-r_{l}^{2}}} r d r$

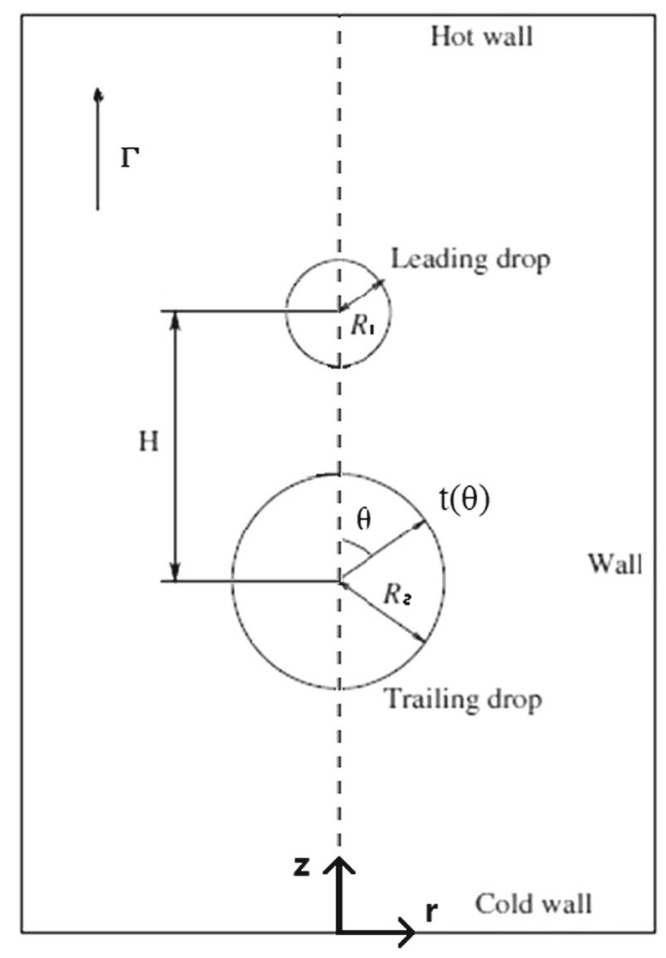

Fig. 4 The schematic diagram of the two drops migration 
Therefore, an algebra layer analysis method is applied to reconstruct distribution of the refractive index change in the liquid field. The integral equations of the optical path difference are discretized into the algebraic equations.

Let

$A_{i l} \Delta r=\int_{r_{i}}^{r_{i+1}} \frac{r d r}{\sqrt{r^{2}-r_{l}^{2}}}$

Then, the following summation equation can be used to replace the integral equation:

$\delta_{l}=2 \Delta r \sum_{i=1}^{k-1} A_{i l} \Delta n_{i}(r)$

The distribution of the refractive index change resulting from the drops migration could be calculated in turn from the outer to centre. Then, the perturbed temperature distribution in the continuous phase is obtained.

The problem of reconstruction of the axisymmetric field has been solved many years ago. However, the physical model of this paper is a square liquid pool, which is different from the axisymmetric field. Therefore, the actual temperature field is a coupling field. For decoupling the actual temperature field $\mathrm{T}_{\mathrm{a}}$, it is divided into two parts, the distribution of the background temperature field $T_{b}$ and the perturbed temperature field $T_{p}$. These two parts need to be calculated, respectively. The distribution of the background temperature without the drop migration is a two-dimensional field, which is obtained by the two-dimensional reconstruction. The distribution of the perturbed temperature resulting from the drops migration is an axisymmetrical field, which is obtained by the onion-peeling algorithm. Finally, the actual temperature distribution in the continuous phase can be obtained and expressed as follows:

$T_{b}+T_{p}=T_{a}$

In this paper, on the basis of the two-dimensional field, the key problem of the coupling reconstruction of the twodimensional field and the axisymmetric field is solved, and the actual temperature distribution in the continuous phase is obtained.

\section{Experimental Results and Analysis}

After the temperature field steady state conditions are achieved, a small leading drop of $3.70 \mathrm{~mm}$ in diameter is injected into the cell first, and then the large trailing drop of $6.94 \mathrm{~mm}$ in diameter is injected later. The radius

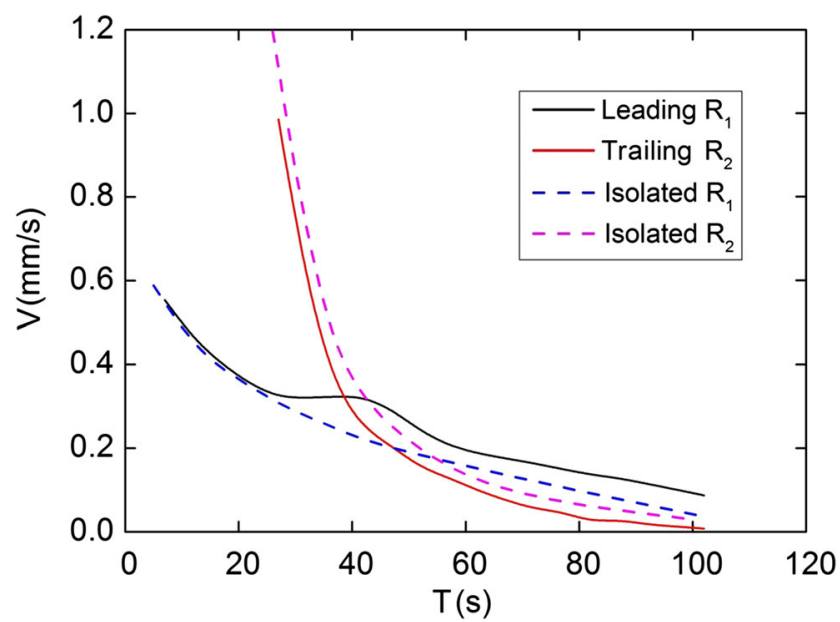

Fig. 5 Comparison of two drops velocities with the isolated drops velocities

ratio of the leading drop to the trailing drop is $\Lambda=0.5$, and the dimensionless initial distance $\mathrm{S}_{0}=2.7$. For the leading drop, Reynolds number is 0.166 , and Marangoni number is 50.5. For the trailing drop, Reynolds number is 0.585 , and Marangoni number is 177 . The experiment results comparison of two drops velocities with the isolated drops velocities are depicted in Fig. 5. When two drops migrated together, it is different from the isolated drops migration. For the leading drop, after inserting the trailing drop behind the leading drop, the leading drop migration velocity is found to be larger than that of the isolated drop. Moreover, there is a velocity fluctuation from $t=30 \mathrm{~s}$ to $\mathrm{t}=50 \mathrm{~s}$. When the distance of separation between the drops becomes larger, the velocity fluctuation disappears. For the trailing drop, the drop migration velocity is found to be less than that of the isolated drop.

Figure 6 shows the time evolution of the distance between centres. It decreases before $t=38 \mathrm{~s}$ because the

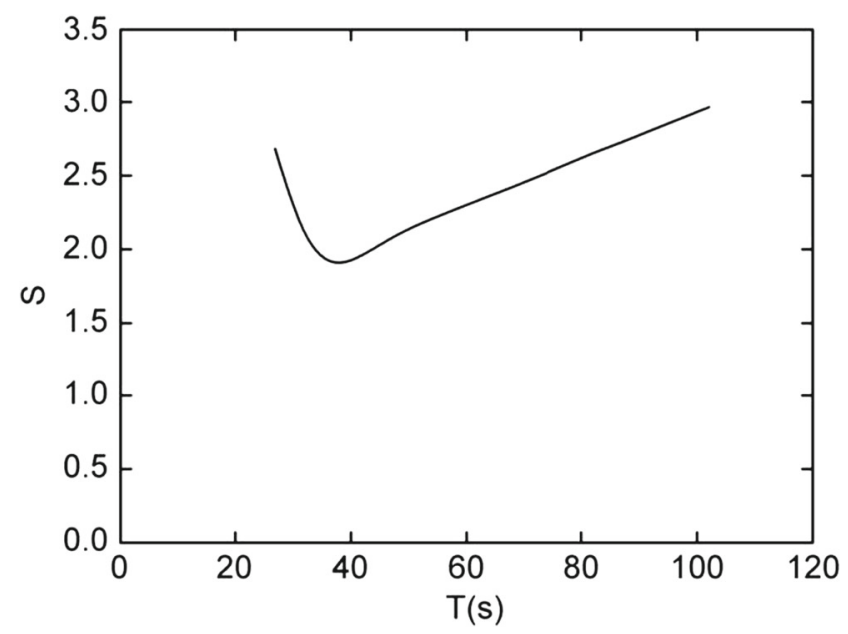

Fig. 6 Distance between centres S 
Fig. 7 Holograms of the drop migration at different times
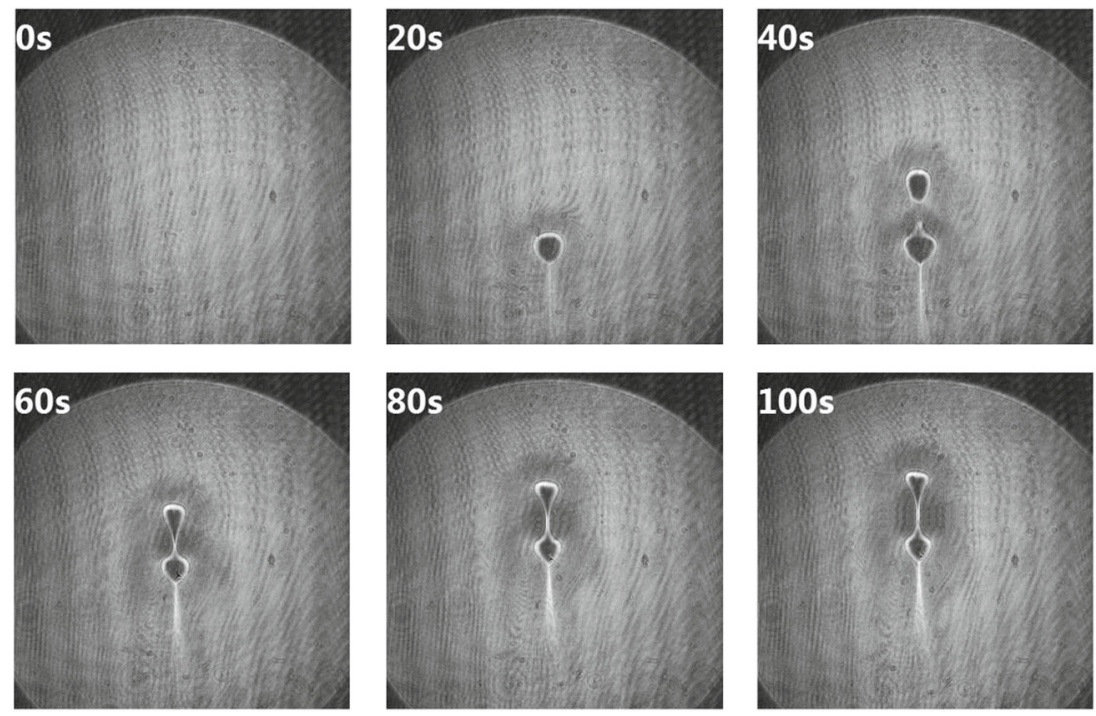

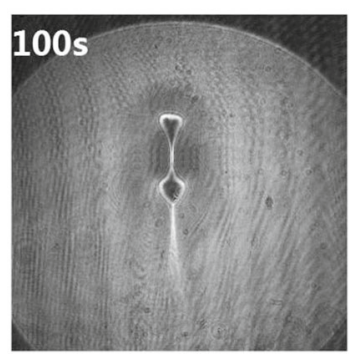

trailing drop is faster than the leading drop, but increases after $\mathrm{t}=38 \mathrm{~s}$ because the trailing drop is slower than the leading drop. The distance of separation between the drops decreases first and then increases with the time whereas if each drop is to move as if it is isolated, the trailing drop would overtake the leading drop.

The above phenomenon can be explained by temperature field around the drops. The interfacial tension gradient drove the drop thermocapillary migration. Then, the larger interface temperature gradient resulted in a greater driving force, and a faster migration velocity. According to the YGB theory, the larger the drop, and the faster it is migrating. However, the migration velocity is different between the two drops migration together and the isolated drop for the same size drop. The migration of two drops exists interaction which has an influence on temperature field, thereby affecting the velocity of the drops. To validate this concept, it is necessary to measure the temperature distribution around the drops during the experiment.

The drops migration interaction is observed through the digital holographic interferometry system. The holograms are separately recorded via CCD after a time interval of $2 \mathrm{~s}$. Figure 7 shows a sequence of holograms at $0,20,40,60,80$ and $100 \mathrm{~s}$, respectively. By numerically reconstructing the object waves, Fig. 8 shows a sequence of the wrapped phase maps at $0,20,40,60,80$ and $100 \mathrm{~s}$, respectively. Using an
Fig. 8 Reconstructed two-dimensional wrapped phase distributions of the drop migration at different times
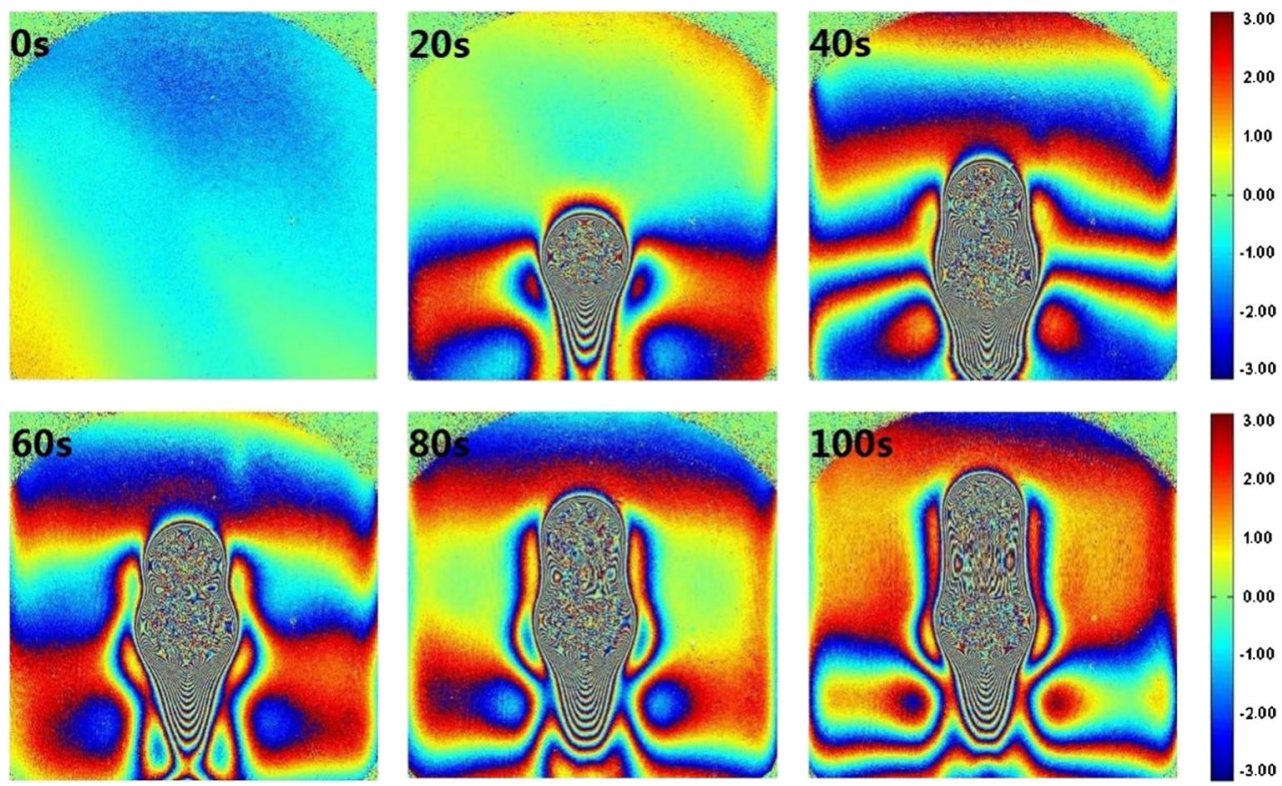
Fig. 9 Actual temperature distribution and isotherms of the actual temperature at $60 \mathrm{~s}$
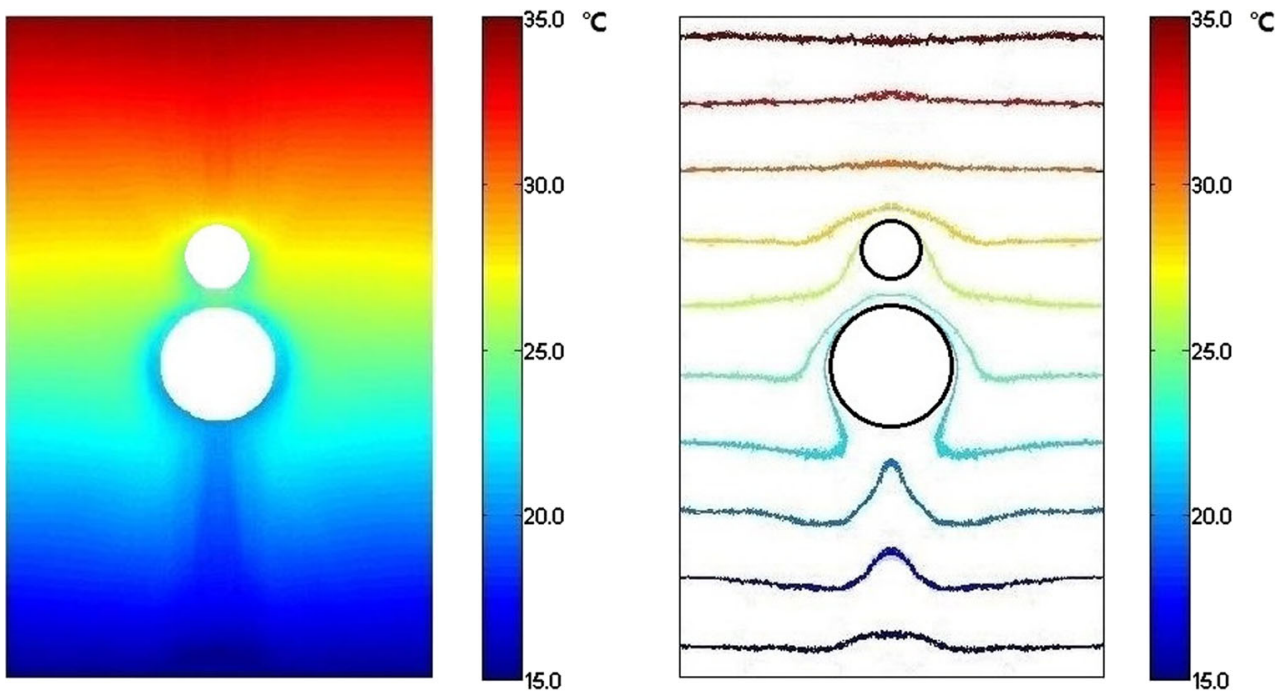

algebra layer analysis of the reconstruction of the refraction index in the axisymmetrical field, the perturbed temperature distribution in the continuous phase is obtained. Combining the perturbed temperature with the initial temperatures, the actual temperature distribution is obtained. Figure 9 shows the actual temperature distribution, as well as the isotherms of the actual temperature at $60 \mathrm{~s}$. The isotherms of the actual temperature, as illustrated in Fig. 9, are neither straight nor vertically symmetrical, but instead wrap around the interface. The drops are colder than the continuous phase liquid, and the thermal wakes existed behind the drops. The trailing drop is inside the region of the thermal wake of the leading drop. In this thermal wake, the temperature field had changed, and the drops migration are affected. The temperature field is one of the most important factors in the interaction between drops.

Figure 10 shows the interfacial temperature distributions of the leading drop and the trailing drop. As shown

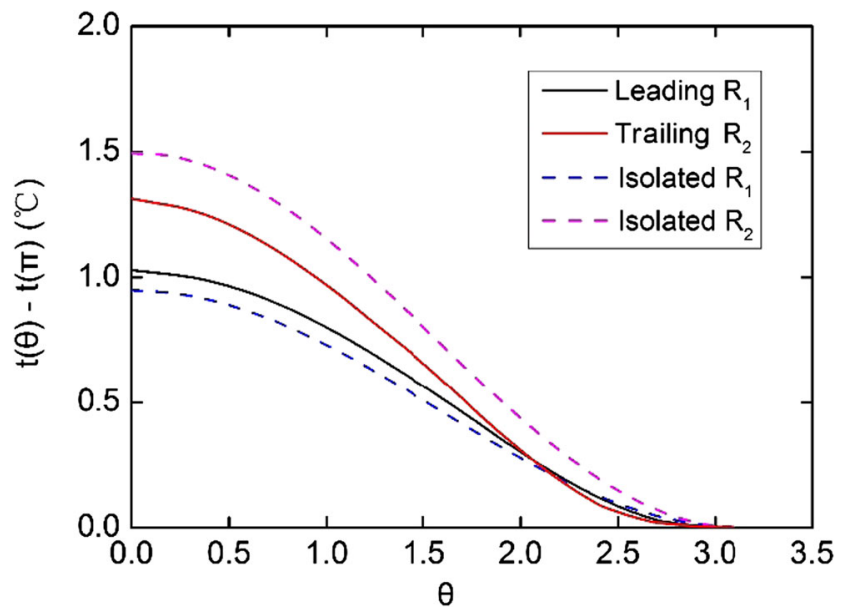

Fig. 10 Interfacial temperature distributions along the drop interfaces at $60 \mathrm{~s}$ in Fig. 10, the maximum of the interfacial temperature distribution of the leading drop is larger than the one of the isolated drop. On the other hand, the maximum of the interfacial temperature distribution of the trailing drop is less than the one of the isolated drop. A larger the maximum of the interfacial temperature distribution of the drop means a larger temperature gradient around the drop, which means a greater driving force, and a faster migration velocity. When two drops migrate together, the drop affects the other drop by perturbing the temperature field around itself. The thermal wake caused by the leading drop lowers the temperature gradient around the trailing drop, and the velocity of the trailing drop is slower than the one of the isolated drop. On the other hand, the migration of the trailing drop accumulated the cold continuous phase around forward stagnation of the trailing drop, which leaded to a large temperature gradient around the leading drop, the velocity of the leading drop is faster than the one of the isolated drop.

Yin and $\mathrm{Li}$ (2015) investigated the axisymmetric thermocapillary migration of two spherical drops by using the front-tracking method. As expected, they found that the thermal wake behind the leading drop plays an important role in drop interaction. In the numerical study, it has been observed that the thermal wake caused by the leading drop lowers the temperature gradient around the trailing drop, and the trailing drop moves more slowly than the isolated drop. Also, they found that the accumulated isotherms around the head of the trailing drop leads to a large temperature gradient around the leading drop, so the leading drop moves faster than if isolated.

In order to consider the influence of the dimensionless initial distance between the drop centres $S_{0}$ to the drop migration, three values of $S_{0}$ are analysed: 2.2, 2.7 and 3.1. 
(a)

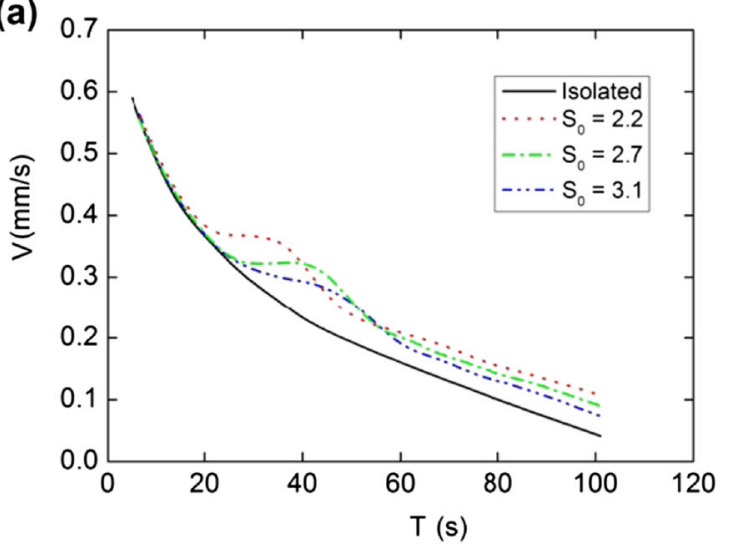

(b)

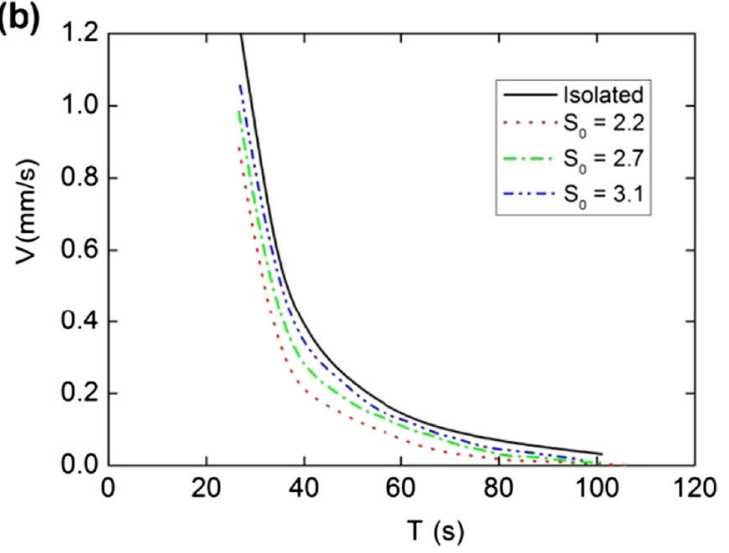

Fig. 11 Drop velocities with different $\mathrm{S}_{0}$

As shown in Fig. 11a and $b$, the variance of $S_{0}$ had an influence on the migrating drop velocities. It should be noted that in regards to the trailing drop, the trailing drop is not injected at the same time in the experiment of three values of $\mathrm{S}_{0}$. To be convenient for comparison, the curves of the trailing drop are moved to the same start time. For the leading drop (Fig. 11a), the velocity of the leading drop is faster than that of the isolated drop for the three values of $\mathrm{S}_{0}$. Figure 11a also shows that when $\mathrm{S}_{0}$ is small, the velocity of the leading drop is fast. By contrast, for the trailing drop (Fig. 11b), the velocity of the trailing drop is slower than that of the isolated drop for the three values of $S_{0}$. Figure $11 \mathrm{~b}$ also shows that when $S_{0}$ is small, the velocity of the trailing drop is slow. When $S_{0}$ is small, the interaction between the drops is obvious. On the contrary, when $\mathrm{S}_{0}$ is large, the interaction between the drops is small, and the migration process of two drops is close to that of the corresponding isolated ones.

Figure 12 shows the time evolution of the distances with different $\mathrm{S}_{0}$. The distance of separation between the drops decreases first and then increases with the time.

Figure $13 \mathrm{a}$ and $\mathrm{b}$ show the interfacial temperature distribution of the leading drop and the trailing drop with different $S_{0}$ at $60 \mathrm{~s}$. For the leading drop (Fig. 13a), the maximum of the interfacial temperature distribution of the leading drop is larger than the one of the isolated drop for the three values of $S_{0}$. Figure 13a also shows that when $\mathrm{S}_{0}$ is small, the maximum of the interfacial temperature distribution is large. The maximum of the interfacial temperature distribution decreased with the increasing the values of $\mathrm{S}_{0}$ for the leading drop. The larger the maximum of the interfacial temperature distribution of the drop is, the larger the temperature gradient around the drop is, and the faster the drop migrated. Therefore, the velocity of the leading drop is faster than that of the isolated drop, and the velocity of the leading drop with small $S_{0}$ is faster than the one with the large $S_{0}$. For the trailing drop
(Fig. 13b), the maximum of the interfacial temperature distribution of the trailing drop is less than the one of the isolated drop for the three values of $S_{0}$. Figure $13 \mathrm{~b}$ also shows that when $S_{0}$ is small, the maximum of the interfacial temperature distribution is small. The maximum of the interfacial temperature distribution increased with the increasing the values of $\mathrm{S}_{0}$ for the trailing drop. Therefore, the velocity of the trailing drop is slower than that of the isolated drop, and the velocity of the trailing drop with small $\mathrm{S}_{0}$ is slower than the one with the large $\mathrm{S}_{0}$.

As shown in Figs. 11 and 13, both the velocity and the interfacial temperature distribution curves of the leading and the trailing drop with $\mathrm{S}_{0}=3.1$ are closer to that of the isolated drop than others. When $S_{0}$ is small, the interaction between the drops is obvious. When $\mathrm{S}_{0}$ is large, the interaction between the drops is small, and the migration process of two drops is close to that of the corresponding isolated ones.

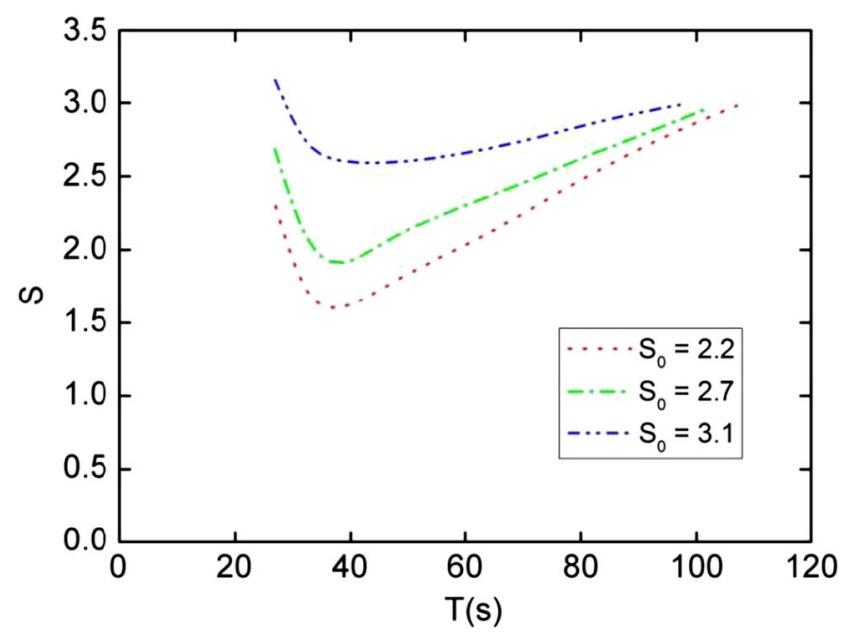

Fig. 12 Time evolution of drop distances with different $\mathrm{S}_{0}$ 
(a)

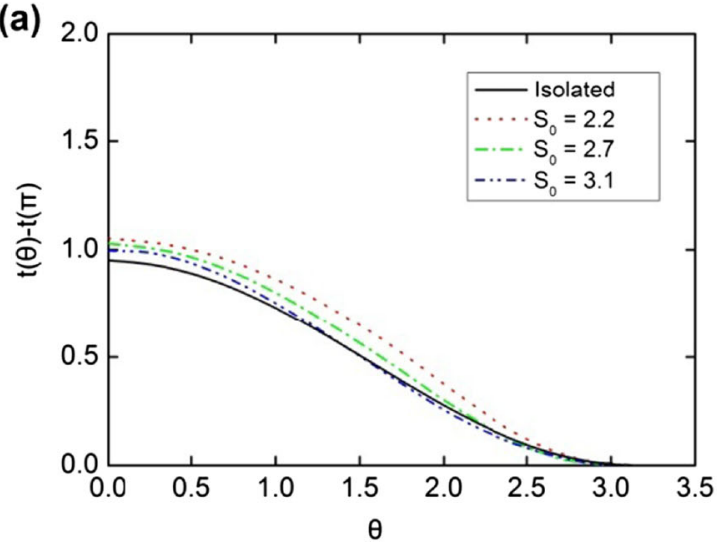

(b)

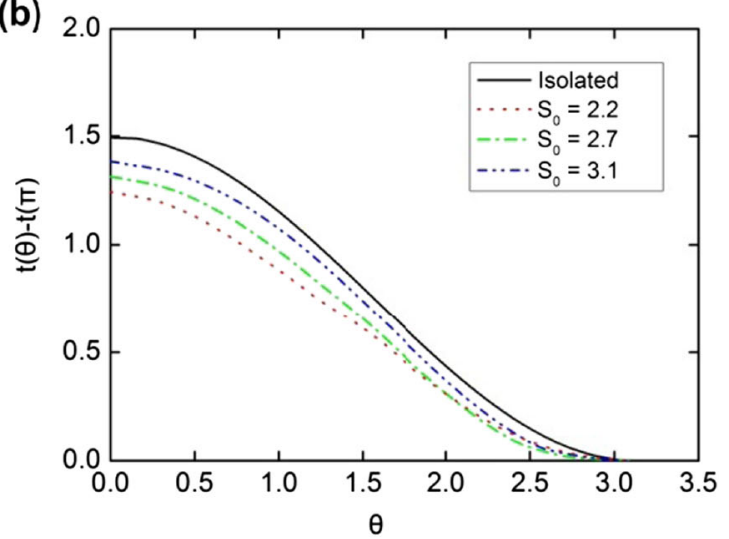

Fig. 13 Interfacial temperature distributions along the drop interfaces with different $S_{0}$ at $60 \mathrm{~s}$

\section{Conclusions}

The thermocapillary-buoyancy migration interaction of the axisymmetric two drops is studied in the present experiment by utilising digital holographic interferometry. During the experimental measurement, the key problem of coupling reconstruction of the two-dimensional field and axisymmetric field is solved, and the temperature distribution around the drops, as well as on the interface of a drop, during migration are visualised and provided quantitatively. Then, the coupling between the temperature and velocity of the migration interaction of drops is studied.

When two drops migrated together, the drop affected the other drop by perturbing the temperature field around itself. For the leading drop, the migration of the trailing drop accumulated the cold continuous phase around forward stagnation of the trailing drop, which leaded to a large temperature gradient around the leading drop. Therefore, the velocity is faster than the one of the isolated drop, and the maximum of the interfacial temperature distribution is larger than the one of the isolated drop. For the trailing drop, the thermal wake caused by the leading drop lowered the temperature gradient around the trailing drop. Therefore, the velocity is slower than the one of the isolated drop, and the maximum of the interfacial temperature distribution is less than the one of the isolated drop. The influence of the leading drop on motion the trailing drop is more significant than that of the trailing drop on the motion of the leading drop.

The influence of the dimensionless initial distance between the drop centres to the drop migration is discussed in detail in this study. When $\mathrm{S}_{0}$ is small, the interaction between the drops is obvious. When $\mathrm{S}_{0}$ is large, the interaction between the drops is small, and the migration process of two drops is close to that of the corresponding isolated ones.
Acknowledgments This research study is funded by the National Natural Science Foundation of China (11372328), by the Strategic Priority Research Programme on Space Science, the Chinese Academy of Sciences: SJ-10 Recoverable Scientific Experiment Satellite (Grant No. XDA04020405 and XDA04020202-05), and by China Manned Space Engineering program. The authors wish to thank the team led by Professor Jianlin Zhao of Northwestern Polytechnical University for providing assistance in using the digital holographic interferometer.

\section{References}

Anderson, J.L.: Droplet interactions in thermocapillary motion. Int. J. Multiphase Flow 11, 813-824 (1985)

Balasubramaniam, R., Lacy, C.E., Woniak, G., Subramanian, R.S.: Thermocapillary migration of bubbles and drops at moderate values of the Marangoni number in reduced gravity. Phys. Fluids 8, 872-880 (1996)

Barton, K.D., Subramanian, R.S.: The migration of liquid drops in a vertical temperature gradient. J. Colloid Interf. Sci. 133, 211-222 (1989)

Dasch, C.J.: One-dimensional tomography: a comparison of Abel, onion-peeling, and filtered backprojection methods. Appl. Opt. 31, 1146-1152 (1992)

Duan, L., Kang, Q., Sun, Z.W., Hu, L., Cui, H.L., Lin, H., Li, G.P.: The real-time Mach-Zehnder interferometer used in space experiment. Microgravity Sci. Technol. 20, 91-98 (2008)

Frolovskaya, O., Nir, A., Lavrenteva, O.M.: Stationary regimes of axisymmetric thermal wake interaction of two buoyant drops at low Reynolds and high Peclet number. Phys. Fluids 18, 072103 (2006)

Hadland, P.H., Balasubramaniam, G., Wozniak, R., Subramanian, R.S.: Thermocapillary migration of bubbles and drops at moderate to large Marangoni number and moderate Reynolds number in reduced gravity. Exp. Fluids 26, 240-248 (1999)

Hähnel, M., Delitzsch, V., Eckelmann, H.: The motion of droplets in a vertical temperature gradient. Phys. Fluids A 1, 1460-1466 (1989)

Kang, Q., Duan, L., Hu, W.R.: Experimental study of surface deformation and flow pattern on buoyant-thermocapillary convection. Microgravity Sci. Technol. 15, 19-24 (2004)

Kang, Q., Hu, L., Huang, C., Cui, H.L., Duan, L., Hu, W.R.: Experimental investigations on interaction of two drops by thermocapillary-buoyancy migration. J. Heat Mass Transf. 49, 2636-2641 (2006) 
Kang, Q., Cui, H.L., Hu, L., Duan, L.: On-board experimental study of bubble thermocapillary migration in a recoverable satellite. Microgravity Sci. Technol. 20, 67-71 (2008)

Keh, H.J., Chen, S.H.: The axisymmetric thermocapillary motion of two fluid droplets. Int. J. Multiphase Flow 16, 515-527 (1990)

Keh, H.J., Chen, S.H.: Droplet interactions in axisymmetric thermocapillary motion. J. Colloid Interf. Sci. 151, 1-16 (1992)

Lavrenteva, O.M., Nir, A.: Axisymmetric thermal wake interaction of two drops in a gravity field at low Reynolds and high Peclet numbers. Phys. Fluids 15, 3006-3014 (2003)

Loewenberg, M., Davis, R.H.: Near-contact thermocapillary motion of two non-conducting drops. J. Fluid Mech. 256, 107-131 (1993)

Nas, S., Tryggvason, G.: Thermocapillary interaction of two bubbles or drops. Int. J. Multiphase Flow 29, 1117-1135 (2003)

Nas, S., Muradoglu, M., Tryggavson, G.: Pattern formation in thermocapillary migration. J. Heat Mass Transf. 49, 2265-2276 (2006)

Rashidnia, N., Balasubramaniam, R.: Thermocapillary migration of liquid droplets in a temperature gradient in a density matched system. Exp. Fluids 11, 167-174 (1991)
Xie, J.C., Lin, H., Han, J.H., Hu, W.R.: Drop migration of middle Reynolds number in a vertical temperature gradient. Microgravity Sci. Technol. 9, 95-99 (1996)

Yin, Z.H., Li, Q.H.: Thermocapillary migration and interaction of drops: two non-merging drops in an aligned arrangement. J. Fluid Mech. 766, 436-467 (2015)

Yin, Z.H., Chang, L., Hu, W.R., Gao, P.: Thermocapillary migration and interaction of two nondeformable drops. Appl. Math. Mech. 32, 811-824 (2011)

Young, N.O., Goldstein, J.S., Block, M.J.: The motion of bubbles in a vertical temperature gradient. J. Fluid Mech. 6, 350-356 (1959)

Zhang, Y.Y., Zhao, J.L., Di, J.L., Jiang, H.Z., Wang, Q., Wang, J., Guo, Y.Z., Yin, D.C.: Real-time monitoring of the solution concentration variation during the crystallization process of protein-lysozyme by using digital holographic interferometry. Opt. Express 20, 18415-18421 (2012)

Zhang, S.T., Duan, L., Kang, Q.: Experimental research on thermocapillary migration of drops by using digital holographic interferometry. Exp. Fluids 57, 1-13 (2016)

Zhou, H., Davis, R.H.: Axisymmetric thermocapillary migration of two deformable viscous drops. J. Colloid Interf. Sci. 181, 60-72 (1996) 